\title{
A Material Handling System Selection Model for Automated Manufacturing
}

Jen Shang

The Joseph M. Katz Graduate School of Business, University of Pittsburgh, Pittsburgh, PA 15260, USA

Abstract: This paper proposes a two-phase method for the evaluation and selection of material handling systems. At phase one, the analytical hierarchy process (AHP) is applied to narrow the large number of output evaluations down to three aggregate measures. At phase two, data envelopment analysis (DEA) is applied to determine the most efficient system. Since multiple efficient units are observed in the basic DEA model, a modification of the original model is necessary to make DEA a normative tool. We add the cross efficiency method and the goal programming procedure to DEA, and finally select the best material handling system.

Keyword: Material Handling Systems, AHP, DEA, Engineering Economy

\section{Introduction .}

- Due to its impact on the cost and efficiency of operations, design and selection of a material handling system (MHS) is one of the most important and challenging tasks a planner faces in automated manufacturing. Several articles have addressed the material handling equipment selection problem (Webster and Reed, 1971; Hassan et al. 1985; Shelton and Jones, 1987). However, majority of the studies have focused on cost minimization. None has dealt with the qualitative, non-quantifiable aspect of the problem. This research emphasizes multiple criteria decision making in MHS selection.

We present a systematic model for MHS selection. The proposed procedure consists of two modules. The first module uses the analytic hierarchy process (AHP) and engineering economic analysis to evaluate the outputs and inputs of each alternative equipment in detail. In the second module, data envelopment analysis (DEA) is employed to integrate all aspects of the measuring criteria. A unique final solution is obtained by developing the cross efficiency matrix through goal programming.

In the following, we will first discuss the inputs and outputs associated with material handling systems. The theory and application of DEA for MHS selection is detailed in Section 3. Section 4 discusses and summarizes the results of this research.

\section{The Measuring Criteria}

Before the AHP technique can be applied, it is necessary to carefully study the factors that influence the selection of the MHS. In addition to the cost factors, the qualitative (intangible) benefits, the quantitative (tangible) benefits, and the vendor's condition must be taken into account and assessed for importance. Qualitative factors are elements that could affect the decision but are generally difficult to quantify. It is important to employ a systematic approach to analyze these factors objectively, consistently, and accurately. This leads us to adopt 
the AHP methodology (Saaty, 1980), a hierarchical multiattribute decision aid.

\subsection{Measuring the Qualitative Benefits}

The qualitative benefits of MHS are tied to the manufacturing operation, and therefore vary from one firm to another. It is usually difficult to place a monetary value on these factors, but they do have a significant effect on the overall performance. Figure 1 shows an AHP based benefit hierarchy. The highest level is the goal. The second level presents the criteria that affect the decision maker's judgement. The third level lists the subcriteria that affect the assessment of each criteria, while the fourth level identifies alternatives. We discuss each qualitative factor below.

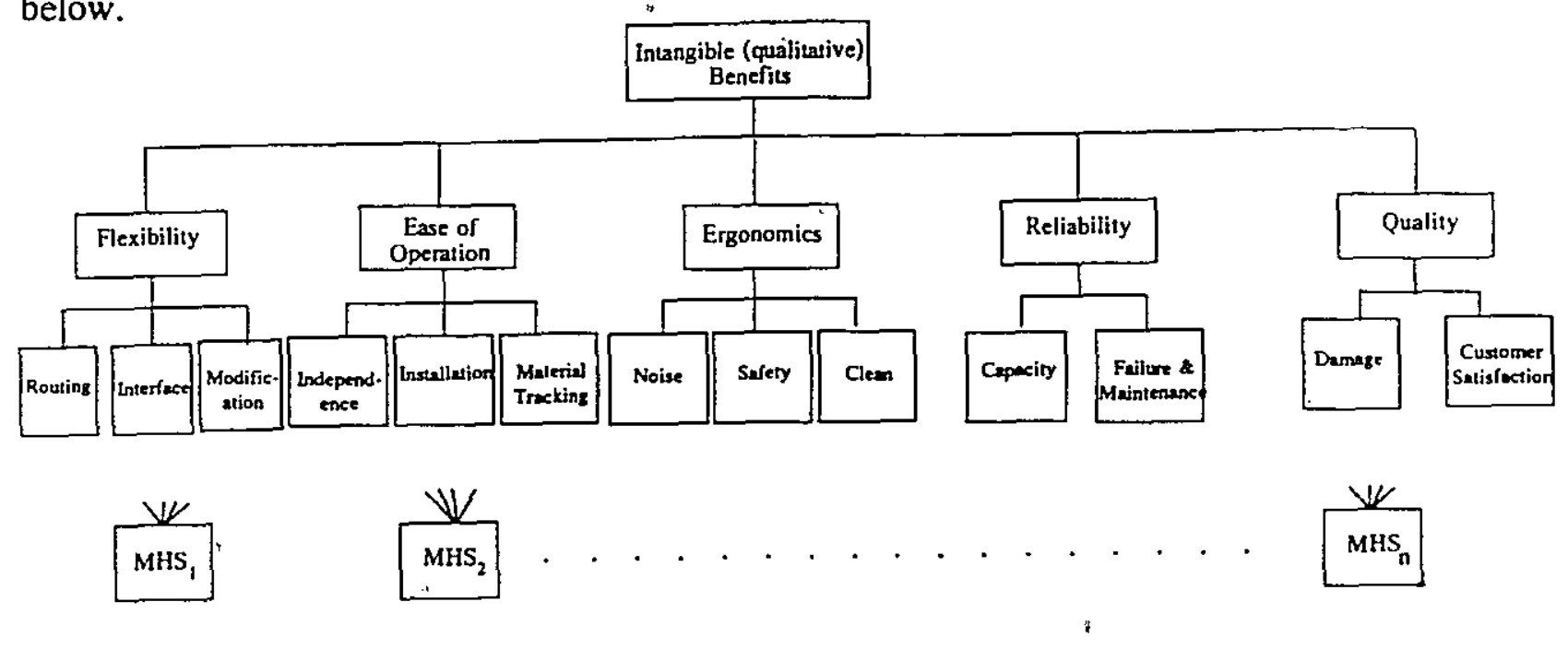

Figure 1. Qualitative Benefits of MHS

\subsubsection{The Qualitative Criteria}

(A) Flexibility.

Flexibility is the ability of the system to respond effectively to change. It can be divided into three categories. (i) Routing flexibility. It is the ability to transport a workpiece from a given workstation to any of several other workstations. Conveyors have no route flexibility due to its operation between two fixed points. An automated guided vehicle (AGV) has a high degree of routing flexibility since a workpart can be picked up and placed at any desired location. (ii) Interface with other equipment. Some MHS operate efficiently with other automated and computer-controlled systems, others are hard to adapt. For example, AGVs can provide a link between different cells of automation within the plant, thereby integrating the overall operation. (iii) System modification. Design change accommodation includes expansion, upgrade and change of speed. When material movement needs to be changed or plant size varies, a good MHS could be expanded or modified quickly and at low cost (Material Handling Institute, Inc. 1983).

(B) Ease of operation. 
Good MHS allow loading and unloading of each station independently of other stations rather than limiting the machine to the speed of the MHS like conveyors do. In terms of installation, AGV can be installed more quickly and economically than most other material handling systems (Hammond, 1986). If the existing floor is suitable, AGVs can also be installed in existing buildings with a minimum of interference to ongoing operations. Good MHS can also identify the material with the device carrying it, providing there is a link for real-time tracking. This in turn reduces time-lag in updating information and makes real-time control possible.

(C) The ergonomic impact.

Some MHS, like AGVs, require very little energy to operate. It is very clean and the vehicles are very quiet, which improves the working environment in the plant. Good MHS also improve the safety and housekeeping in the plant. Usually, the floor conveyors tend to accumulate waste and contaminants. Floors that are dirty or have extremely uneven surfaces are not suitable for AGVs.

(D) Reliability of the system.

Good MHS provide reliable capacity. For example, in an AGV system, if one vehicle requires maintenarice, the other vehicles will not be impaired due to their independence of each other. When a disabled vehicle is taken away from the system, a spare vehicle can be used to retain its capacity. However, some advanced material handling equipments are more complicated than others. Accordingly, they may be failure prone and require more maintenance.

(E) Quality.

A good MHS transports material in a controlled manner, and hence reduces product damage. Product quality is improved due to less damage in handling. For example, in AGVs the damage is reduced because the vehicles travel on a predetermined route and contain safety features that make it virtually impossible to collide with racks and other obstacles. As a result, it leads to less scrap, less repair, and improved customer satisfaction.

\subsubsection{Assessment}

After constructing the hierarchy for the AHP model, we proceed to pairwise comparisons. It is relatively easy to consider each qualitative factor pair by pair instead of considering them all at one time. After the relative importance of each criteria and subcriteria are assessed, we then indicate our preference for each of the alternative relative to each subcriteria. Given the information on relative importance, AHP synthesizes the information and provides a priority ranking of the alternatives in terms of their qualitative performance.

\subsection{Measuring the Quantitative Benefits}

Several tools exist for estimating the tangible retum that can be achieved from an investment in manufacturing equipment. These tools include mathematical programming, queuing analysis, and computer simulation. Simulation provides a rational basis for quantifying the 
tangible factors. It may be performed by an outside consultant, in-house personnel, or the MHS vendor.

Three major types of information can be derived from the MHS simulation. They are time related outputs, number related outputs, and utilization oriented outputs (Koff and Boldrin, 1985).

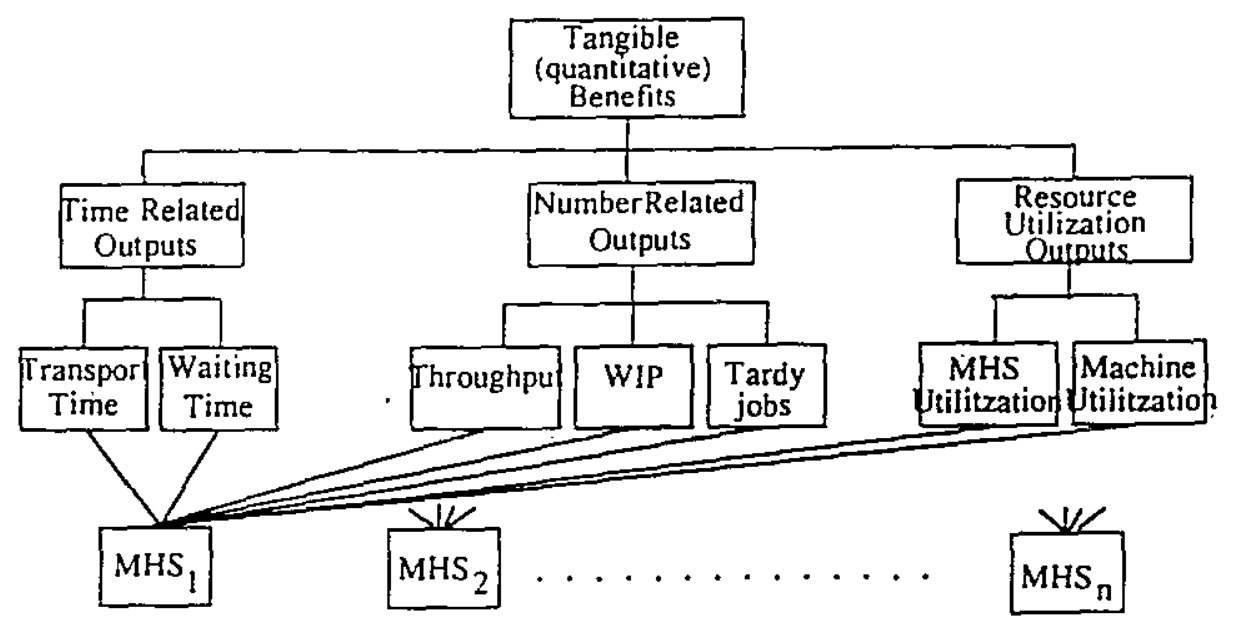

Figure 2. Quantitative Benefits of MHS

\subsubsection{Quantitative Criteria (see Figure 2)}

(A) Time related outputs.

Manufacturing lead time has significant bearing on overall production costs. In a typical manufacturing environment, the flow time consists of setup time, processing time, waiting time and moving time. The processing time and setup time (assuming sequence independent) are usually deterministic, and do not affect the overall flow time. Waiting time and moving time constitute the majority of the manufacturing lead time. They are highly dependent on the system design and operation. Transport time is the time needed to move workpieces from one workstation to another. The best material handling is no material handling and the ideal move time is zero. Queuing time is the time workparts spend waiting for resources such as machines and MHS. The waiting time can be reduced if machines and MHS are properly selected and adequately operated.

(B) Number related outputs.

Throughput, number of tardy jobs, and work-in-process belong to this category. Throughput is usually expressed as the number of parts produced per hour or day. It could be characterized as the average number of units departing from the unloading station hourly or daily. Work-in-process is the amount of semifinished goods or subassemblies residing on the shop floor in various stages of processing. WIPs are regarded as bad since they are the evidence of poor coordination and operation of the manufacturing system. With good MHS, inventory could be reduced through accurate tracking of the use and transfer of material. With better scheduling, an on-line interface to production and inventory control become possible. Through the efficient transfer mechanism, the WIPs are delivered quickly and the number of tardy jobs are reduced. 


\section{(C) Utilization Oriented Outputs}

Two types of utilization measures are considered. They are MHS utilization and machine utilization. The MHS utilization rate discloses the adequacy of the MHS capacity. Too many (low utilization) or too few (high utilization) capacities are considered to be inadequate since too many cause waste and traffic congestions; while too few lead to long job waiting time for the MHS device.

High machine utilization means high manufacturing efficiency. Yet, this is favored only when the machines are used to produce items mandated by current orders. Producing unassured demand for the future results in high machine productivity and accumulates huge inventories. Building up unrequested products ties up the cash in raw material and components, leads to the risk of inventory obsolescence, and increases the holding and managing cost. However, when production is made to order, high machine utilization is desired. An efficient MHS helps increase the machine utilization by delivering the right material to the right workstation at the right time.

\subsubsection{Assessment}

The procedure for criteria weight establishment is the same as those described in Section 2.1.2. After the weights are determined for each criterion and subcriterion, we have to evaluate the alternatives. In many AHP applications, the decision makers abandon the original quantitative information and make subjective judgements over the quantifiable data. We believe subjective judgements should be used only when credible data reflecting the performance of the alternative are not available. When alternatives are measured on a quantitative scale, the values residing on the pairwise comparison matrix should equal the corresponding numerical ratios of each pair. The usual approach of eliciting response from the decision makers should not be pursued. With the direct numerical comparison method, we can better preserve the original informing power of the data. If the measuring criterion has the-smaller-the-better characteristic, the inverse ratios of the numerical data are used in the comparison matrix. In case the importance of the numbers are interpreted in a nonlinear way, a nonlinear function, such as exponential or logarithm, should be used to convert the original data into a nonlinear form before any comparison matrix is constructed. After all alternatives are rated and the criteria weights are determined, the composite score is derived as usual.

\subsection{Vendor Support, Cooperation and Services (See Figure 3)}

To successfully implement a material handling system it is important that a strong working relationship between the user and the vendor be developed. In the beginning, vendors should undertake initial steps by exploring the design of a system that can satisfy each user's objectives and production needs. It is essential that the chosen vendors stay in business for some time so the user can receive the essential service and upgrade the system when necessary. The supplier's reputation and financial status offer possible indicators of their commitment to stay. Vendor support includes the ability of the vendor to integrate the proposed MHS with other system components. It also involves the vendor's commitment to the specific MHS. It is also important to obtain a list of installations that the supplier has provided, interview the users and get their reactions. The vendor's familiarity with the firm's floor operations, responsiveness, 
training program, financing terms, warranty, and the level of expertise necessary to design, specify, and install the system are all important in vendor selection.

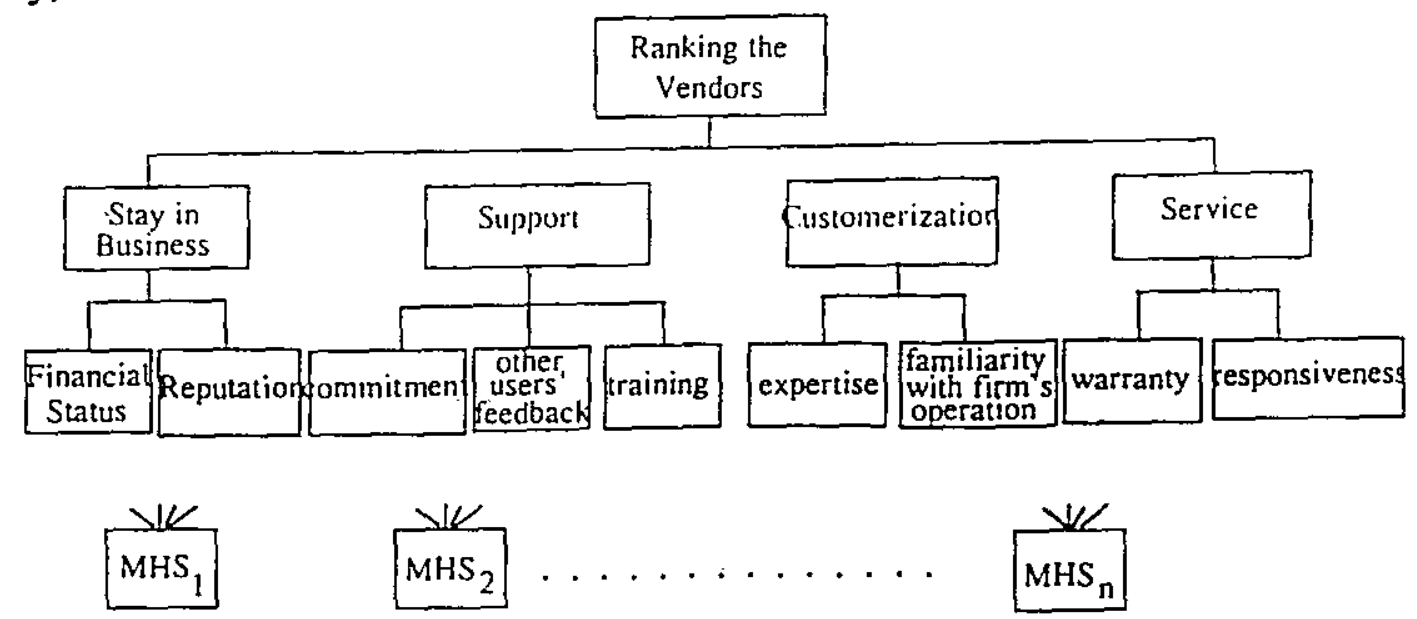

Figure 3. The Characteristics of MHS Vendors

\subsection{Resource Requirements}

Inputs of an MHS is the investment needed to ensure the operation of a system. The resources necessary to implement and operate a material handling system include the capital outflow and.space requirements. A number of methods and criteria exist for measuring the cost of investments. Two commonly used approaches are present worth and annual worth methods. The present worth method converts all cash flows to a single sum equivalent at time zero using the current interest rate. The annual worth method converts all cash flows to an equivalent uniform series of cash flows over the planning horizon using the current interest rate (White et al. 1989). These two methods are related by a constant multiplier, (A/P, i, N) (described later), and are therefore equivalent in measure. The choice of method depends on the preference of users. In this article, we choose to spread the cost out uniformly over the planning horizon, so the annual worth method is used to calculate the annual cost.

\section{(A) Annual Cost.}

Costs of MHS investments include the following three items: (i) Equipment cost, which comprises the purchasing of the equipment and auxiliary components, and installation. (ii) Training cost, which is the cost of training the line-workers as well as the management. (iii) Operating cost, which includes the maintenance and labor cost. When advanced MHS is used, substantial operating savings result due to reduction in the number of material handling personnel and the necessary coordination between them. However, the maintenance cost may increase.

The annual cost of investment can be computed as follows. Consider an alternative MHS requiring a lump-sum investment $M$, training cost $T$, and annual operating cost $Q$. And assume it has a salvage value $S$ at the end of $N$ years. At annual interest rate $i \%$, the annual cost can be calculated as: $A C=(M+T)^{*}(A / P, i \%, N)-S^{*}(A / F, i \%, N)+Q$. Here, the $(A / P, i \%, N)$ is used to convert present value $P$ to annuity $A$ for the planning horizon $N$ under interest rate $i$. It is equal to $\left(i(1+i)^{N}\right) /\left((1+i)^{N}-1\right)$ and is named the capital recovery factor. The sinking fund factor, $(A / F, i \%, N)$, is defined as $i /\left((1+i)^{*}-1\right)$, which is the multiplier for converting future 
value $F$ to annuity $A$.

(B). Floor space.

Extra space costs money to attain. Valuable floor space should be used for production instead of being used for installation of material handling equipment. In addition to the direct floor space occupation by the MHS, extra floor space may be needed indirectly due to inferior inventory control, slow transporter response and increase in online stock. Increase in floor space requirements has forced some companies to expand their plant or to extend their operations to a second factory.

By now we have examined the costs (inputs) and benefits (outputs) associated with the alternative MHS. We can now synthesize the cost hierarchy, and form the benefit to cost ratio for each alternative, so it reveals the alternative with the largest ratio. This approach has been demonstrated to be effective in decision making (Saaty, 1994). However, in this research, instead of adopting AHP's benefit/cost ratio, we will explore the potential of using DEA as a normative tool for selecting the best investment opportunity.

\section{The DEA Approach for MHS Selection}

\subsection{The DEA Model}

DEA has been demonstrated through numerous applications for analyzing the operational processes. However, in spite of its exposure, the methodology remains unknown to a significant portion of the decision scientists. We will describe it here. DEA is a procedure for measuring relative efficiency in situations where there are multiple inputs and outputs (Charnes, et al. 1978; Boussofiane et al. 1991). The technique is important for measuring productivity. The operating units (alternatives) in DEA are called Decision Making Units (DMUs). The relative efficiency of a DMU is defined as the ratio of its total weighted output to its total weighted input. Charnes et al. (1978) show that the efficiency of a target unit $\mathrm{k}$ can be obtained by solving the model below:

$$
\begin{aligned}
& \operatorname{Max} z_{\mu k}=\frac{\sum_{p=1}^{1} u_{k p} y_{k p}}{\sum_{r=1}^{m} v_{k} x_{k}} \\
& \text { s.t. } \\
& \frac{\sum_{p=1}^{t} u_{b p} Y_{j p}}{\sum_{r=1}^{m} v_{b} x_{j r}} \leq 1, \quad j=1, \ldots, n . \\
& u_{k}, v_{b} \geq 0 . \quad \vee p, r .
\end{aligned}
$$

where $y_{k p}=$ amount of output $p$ from DMU $k$,

$x_{k r}=$ amount of input $r$ from DMU $k$,

$u_{k_{p}}=$ the weight given to output $p$ from the viewpoint of DMU $k$,

$v_{k r}=$ the weight given to input $r$ from the viewpoint of DMU $k$, 


$$
\begin{aligned}
& n=\text { the number of decision making units, } \\
& t=\text { total number of outputs, } \\
& m=\text { total number of inputs }
\end{aligned}
$$

The key feature of this model is that the weights $u_{k p}$ and $v_{k r}$ are treated as unknowns. As demonstrated in P1, each DMU can select any weight vector it wants for its inputs and outputs, given that no weights are negative and that the weights are universal. That is, any DMU should be able to use the same set of weights to evaluate itself and guarantee the resulting ratio not to exceed one (Sexton, 1986). In problem P1, the solution maximizes the efficiency of the targeted unit $\mathrm{k}$. The efficiency of unit $\mathrm{k}$ is either equal to 1 , in which case it is efficient relative to the other DMUs; or it is less than 1 , in which case the DMU is inefficient. The efficiency of each DMU relative to the set can be found by solving a similar model, targeting one DMU at a time.

Problem P1 is a fractional linear program, but can be converted easily into linear form so that the methods of linear programming can be applied. The linear programming version of the model is as follows:

(P2)

$$
\begin{aligned}
& \operatorname{Max} z_{\mu}=\sum_{p=1}^{1} u_{\phi} Y_{\Delta} \\
& \sum_{r=1}^{m} v_{k} x_{k r}=1 \\
& \sum_{p=1}^{b} u_{k p} y_{j p}-\sum_{r=1}^{m} v_{b} x_{j r} \leq 0, \quad f=1, \ldots, n \text {, } \\
& u_{\infty} \geq 0, \vee p, \\
& v_{k r} \geq 0, \quad \forall r
\end{aligned}
$$

The objective function has been linearized by recognizing that in maximizing a ratio it is the relative magnitudes of the numerator and the denominator that are important, and not their actual values. The denominator is set equal to a constant (arbitrarily set at 1) and the numerator is being maximized. This model promises advantages which include the absence of need for the assignment of weights on an a priori basis (to reflect the supposed relative importance of various outputs or inputs) when evaluating technical efficiency. This model also eliminates the need to explicitly specify underlying functional relations that are supposed to analytically prescribe the relations between inputs and outputs.

\subsection{Evaluating the Efficiency of Different Material Handling Systems}

In this section, we apply the preceding ideas and form the to-be-minimized (e.g. costs) criteria as inputs, and the to-be-maximized criteria (benefits) as outputs. We consider a set of eight material handling systems whose inputs and outputs are shown in Table 1. All these data sets are artificially generated for the purpose of illustration. Note that outputs are expressed as relative ratios, while the inputs are expressed as absolute values. It is not necessary that all dimensions are expressed in the same measure, since DEA assumes constant returns to scale. Results of the linear programming, (P2), are presented on the rightmost column of Table 1. We found that multiple efficient DMUs exist. Thus, further analysis is necessary to differentiate among all the efficient MHS. 
Table 1. Inputs and Outputs for the Material Handling Systems Inputs

Qutputs

\begin{tabular}{|c|c|c|c|c|c|c|}
\hline DMU & $\frac{\text { Annual Cost }}{(\$ 0.0000)}$ & $\frac{\text { Floor Space }}{\left(000 \mathrm{ft}^{2}\right)}$ & $\begin{array}{l}\text { Intangible } \\
\text { Benefits (\%) }\end{array}$ & $\begin{array}{l}\text { Tangible } \\
\text { Benefits }(\%)\end{array}$ & $\begin{array}{l}\text { Vendor } \\
\text { Supoort (\%) }\end{array}$ & Efficienc \\
\hline 1 & 12.1 & 8.0 & 10.6 & 13.5 & 12.3 & 0.947 \\
\hline 2 & 15.4 & 7.3 & 14.7 & 8.1 & 7.8 & 0.848 \\
\hline 3 & 13.7 & 9.5 & 12.8 & 10.9 & 9.1 & 0.852 \\
\hline 4 & 16.8 & 7.4 & 18.9 & 13.4 & 15.3 & 1.000 \\
\hline 5 & 7.9 & 13.4 & 8.3 & 10.8 & 14.1 & 1.000 \\
\hline 6 & 11.5 & 10.3 & 9.7 & 11.2 & 10.6 & 0.844 \\
\hline 7 & 10.2 & 5.8 & 8.1 & 13.7 & 11.5 & 1.000 \\
\hline 8 & 18.4 & 8.7 & 16.9 & 18.4 & 19.3 & 1.000 \\
\hline
\end{tabular}

\subsection{Targeted Aggression Approach}

The multiple efficient DMUs suggest that DEA is not sufficient in discriminating the best alternative from the others. Sexton (1986) recommend four strategies to alleviate such difficulty. They are: input/output weight restriction, cross efficiency, goal programming, and the clustering method. Extensive discussion of the AHP approach to input/output weight restriction can be found in Shang and Sueyoshi (1994). In this section, we apply the cross efficiency and goal programming procedure to increase DEA's discriminating power. The cross efficiency technique is a peer-evaluation method that subjects each DMU to a range of input-output multipliers. The multipliers are the optimal weights chosen by each DMU. Cross efficiency conveys the information on how the relative efficiency of a DMU is rated by other units. A DMU can rate itself very high by allowing itself to choose any weight vector for the inputs and outputs; however, it can also be rated very low by the majority of other DMUs. This indicates that the rated DMU is a niche player. A niche player excels in a few aspects, but may be inferior in many other dimensions. For example, an MHS may be inexpensive and, as a result, the DEA model gives it an efficiency score of 1 . However, the majority of MHS are superior in other dimensions. Evaluated by the cross efficiency method, a niche player will come out unfavorably on the majority of weights imposed upon it.

To employ the cross efficiency method, we need a unique weight vector from the ranking DMU. Yet, in DEA, the weight vector that maximizes the efficiency of a DMU is not necessarily unique. Thus, the cross-efficiency matrix may not be unique. One resolution to this problem is to force each evaluating $\mathrm{k}$ to choose from among its various optimal weight vectors in order to optimize a secondary goal. Sexton (1986) propose a blanket aggressive method, which minimizes other DMUs efficiency taken as a whole. That is, it minimizes $\frac{1}{(n-1)} \cdot \sum_{j \neq k}^{n} D_{k j}$. Compared to the blanket benevolent formulation, which maximizes the secondary goal, minimization seems appropriate since each material handling system tries to emphasize its supremacy. With the blanket aggressive formulation, the indeterminacy is unlikely, yet it still cannot guarantee uniqueness. To rectify this problem, Doyle and Green (1993) present a targeted aggression method, which guarantees unique cross-evaluations.

In the targeted aggression method, two goals are involved. The primary goal is to maximize the efficiency of DMU $k$, and the secondary goal is to minimize the efficiency of DMU $j$ when the weights generated by DMU $k$ are used. This method differs from the blanket 
aggressive method in that the former targets its aggression at each individual DMU, while the latter blankets one's aggression over the entire set of the remaining DMUs.

The secondary goal should represent a quantity that is to be optimized only in the event that there exists multiple optimal solutions in the original problem, and that should be ignored if there is only a unique optimal solution. When multiple solutions are observed, the cross efficiency of DMU $j$ can be found by:

$$
\begin{aligned}
\text { Min } z_{j k}= & \left(\sum_{p=1}^{t} U_{k p} Y_{j p}\right) /\left(\sum_{r=1}^{m} V_{k r} X_{j r}\right) \\
\text { st. } \quad & \left(\sum_{p=1}^{t} U_{k p} Y_{i p}\right) /\left(\sum_{r=1}^{m} V_{k r} X_{i r}\right) \leq 1, \quad \forall i \neq k \\
& \left(\sum_{p=1}^{t} U_{k p} Y_{k p}\right) /\left(\sum_{Y=1}^{m} v_{k r} X_{k r}\right)=z_{k k} \\
& U_{k p} \geq 0, V_{k r} \geq 0 \quad \forall p, r
\end{aligned}
$$

Table 2 shows the results of the cross efficiency matrix for the illustrated problem. The entry in position $(j, k)$ for $j \neq k$, is the minimum efficiency of unit $j$ derived from the optimal weights of DMU k. For example, entry $(5,5)$ in Table 5 shows that DMU 5 has a self-rated efficiency score of 1 . However, the optimal weight vector is not unique in DMU 5. Among the multiple optimal weights of DMU 5, DMU 1 uses the weight vector that minimizes its own efficiency. We obtain 0.570 for DMU 1 when rated by DMU 5 .

By reading across row $j$ of the cross-efficiency matrix, we learn how $D M U j$ is minimally rated by using the optimal weights from the other DMUs in turn. For example, the mean efficiency in row 1 is .7640 , which is the average efficiency of DMU 1. A DMU with low average efficiency implies that the unit relies on a production technology unlike that of most other DMUs in order to appear efficient (Boussofiane et al. ,1991). On the other hand, DMUs with high efficiency would have chosen similar weights as those selected by other DMUs. Good practice is more likely to be exhibited by relatively efficient units offering high average efficiencies in their associated rows. In Table 2, DMU 4 has the highest average efficiency,

\begin{tabular}{|c|c|c|c|c|c|c|c|c|c|}
\hline \multirow[b]{2}{*}{ Rated } & \multicolumn{9}{|c|}{ Rating MHS } \\
\hline & 1 & 2 & 3 & 4 & 5 & 6 & 7 & 8 & Average \\
\hline \multicolumn{10}{|c|}{ MHS } \\
\hline 1 & 0.947 & 0.779 & 0.946 & 0.519 & 0.570 & 0.947 & 0.714 & 0.691 & 0.764 \\
\hline 2 & 0.784 & 0.848 & 0.784 & 0.580 & 0.284 & 0.784 & 0.392 & 0.482 & 0.617 \\
\hline 3 & 0.852 & 0.831 & 0.852 & 0.478 & 0.372 & 0.853 & 0.485 & 0.432 & 0.644 \\
\hline 4 & 1.000 & 1.000 & 1.000 & 1.000 & 0.510 & 1.000 & 0.595 & 0.891 & 0.875 \\
\hline 5 & 0.999 & 0.934 & 0.999 & 0.243 & 1.000 & 1.000 & 0.341 & 0.362 & 0.735 \\
\hline 6 & 0.844 & 0.750 & 0.844 & 0.369 & 0.516 & 0.844 & 0.460 & 0.464 & 0.637 \\
\hline 7 & 0.973 & 0.706 & 0.972 & 0.547 & 0.632 & 0.973 & 1.000 & 0.863 & 0.833 \\
\hline 8 & 0.952 & 0.817 & 0.951 & 0.761 & 0.588 & 0.952 & 0.746 & 1.000 & 0.846 \\
\hline
\end{tabular}
0.875 . Therefore, we choose MHS 4.

Table 5. Cross Efriciency Matrix for All MHS Using Targeted Aggression Approach 
Despite the usefulness of the above methodology, DEA has received some criticisms. For example, the input and output weights produced by DEA cannot be interpreted as values in the economic sense (Sexton, 1986). A detailed discussion of the theoretical issue is beyond the scope of this article. Our purpose here is to present DEA as a practical method for evaluating alternatives.

\section{Summary and Conclusions}

In this research, we introduce a two-phase method to aid the decision making in MHS selection for automated manufacturing. Decision making in this domain involves multiple dimensions. Thus, the methodology of multicriteria decision making (MCDM) is chosen as the framework of analysis. To choose among the various material handling systems, we adopt DEA methodology for efficiency comparison. Though useful, DEA requires the number of alternatives to be larger than the dimensions, otherwise most alternatives will be efficient (Boussofiane et al. 1991). To ensure that DEA is useful for the encountered problem, we use a mess reduction procedure to aggregate the large number of evaluation criteria and narrow down the dimensions involved. In phase one, we choose AHP to help users assess their preferences and priorities with respect to tangible benefits, intangible benefits, and the vendor-user relationship. The results of these three aggregate measures are used as outputs for the DEA model. In addition, the inputs (resources) needed to attain the benefits of an MHS are identified as the annual cost and floor space requirements. Annual cost, including the equipment cost, training cost and operating cost, is analyzed by the engineering economic analysis.

At phase two, the DEA model is constructed to determine the relative efficiency of each MHS system. Multiple efficient units are often observed. To increase the discriminating power of DEA, we adopt the cross efficiency method. For a perfectly efficient $\left(D_{k k}=1\right) D M U$, its linear program almost surely has multiple optimal solutions, so a secondary goal is needed to derive a unique cross efficiency value for the evaluated DMU. Through the targeted aggression approach, we are able to select the best material handling system.

The contribution of this research is threefold. First, we introduce the DEA technique to the production management area. Here we are not suggesting that the DEA technique is the single best tool for differentiating the potential MHS, but we strongly believe that DEA is an important and powerful tool that can be useful on problems in this domain. Second, using AHP greatly reduces the dimension of DEA. Dimension restriction is a major weakness of DEA. By using AHP, all the relevant data will remain in the decision process. However, only the aggregate values derived from the AHP hierarchy are entered as DEA's inputs and/or outputs. In the past, analysts may have avoided DEA because numerous dimensions are associated with their data. Now, by reducing the input/output measure through AHP, we believe that the number of problems that DEA can tackle will greatly increase. Third, it provides a very comprehensive analysis in MHS selection. We integrate the tangible, intangible, and economical factors into one model. Normally, the engineering economists exclude the consideration of intangible factors; and those who consider the strategic impacts do not incorporate detailed analysis about cost components. The proposed two-phase method combines all the relevant information, and the accuracy of the original numerical data is well preserved in AHP. We believe that this research not only contributes to the MHS literature, but is also general enough to be applicable to many decision making problems. 


\section{REFERENCES}

Boussofiane, A., Dyson, R.G. and Thanassoulis, E. (1991), "Applied Data Envelopment Analysis", European Journal of Operarional Research 52, 1-15.

Charnes, A., Cooper, W.W., and Rhodes, E. (1978), "Measuring the Efficiency of Decision Making Units," European Journal of Operational Research, 1, 429-444.

Doyle, J. and Green, R. (1993), "Data Envelopment Analysis and Multiple Criteria Decision Making," OMEGA, 21/6, 713-715.

Hammond, G. (1986), AGVs at Work: Automated Guided Vehicle Systems, Springer-Verlag, New York.

Hassan, M.M.D., G.L. Hogg, and D.R. Smith (1985), "A Construction Algorithm for the Selection and Assignment of Material Handling Equipment," International Journal of Production Research, 23/2, 381-392.

Koff, .A. and Boldrin, B. (1985), Material Handling Handbook, Wiley Interscience.

Maleki, R.A. (1991), Flexible Manufacturing Systems: the technology and management, Prentice Hall, Inc. Englewood Cliff, New Jersey 07632.

Material Handling Institute, Inc. (1983), Considerations for Planning and Installing Automatic Guided Vehicle Systems, Charlotte, NC, AGVs Document 101, 6-7.

Saaty, T.L. (1980), The Analytic Hierarchy Process, McGraw-Hill Co., New York.

Saaty, T.L. (1994), Fundamentals of Decision Making and Priority Theory, RWS Publications, 4922 Ellsworth Avenue, Pittsburgh, PA 15213.

Sexton, T.R. (1986), "The Methodology of Data Envelopment Analysis," in R.H. Silkman (ed.), in Measuring Efficiency: An Assessment of Data Envelopment Analysis, New Directions for Program Evaluation, 32, San Francisco, Jossey Bass, Inc.

Shang, J.S, and Sueyoshi, T. (1994), "A Unified Framework for the Selection of a Flexible Manufacturing System," European Joumal of Operational Research. (Forthcoming)

Webster, D.B., and R.Jr. Reed (1971), "A Material Handling System Selection Model," AIIE Transactions, 3/1, 13-21.

White, J.A., Marvin, H.A., and Case, K.E. (1989), Principles of Engineering Economic Analysis, John Wiley \& Sons, Inc, New York. 